DOCTRINA

\title{
Sobre la conveniencia de incluir los neuroderechos en la Constitución o en la ley
}

\author{
On the convenience of including neurorights in the Constitution or in the law
}

\author{
Pablo López-Silva \\ Universidad de Valparaíso, Chile \\ Raúl Madrid \\ Pontificia Universidad Católica de Chile
}

\begin{abstract}
RESUMEN Los actuales avances en el campo de las neurotecnologías prometen revolucionar el tratamiento de muchas condiciones médicas. Sin embargo, para algunos, tales avances podrían exacerbar la inequidad social y ofrecer a corporaciones, hackers y Gobiernos internacionales la oportunidad de explotar y manipular le mente humana. Por esto, se ha sugerido durante los últimos años que el desarrollo de cualquier tipo de neurotecnología debería estar acompañado de un suporte normativo que tuviese como objetivo la protección de la privacidad, identidad, agencia y equidad de las personas. A este soporte legal se le ha denominado recientemente neuroderechos. En este artículo nos centramos en si se debiese legislar sobre neuroderechos a la luz del actual desarrollo de la investigación biotecnológica a nivel nacional y mundial. El trabajo no consiste en un análisis del proyecto actualmente en desarrollo, sino en una reflexión sobre la idea misma de legislar sobre el tema.
\end{abstract}

PALABRAS CLAVE Neurotecnología, neuroderechos, privacidad, agencia, neuroprotección.

ABSTRACT Current advances in neurotechnology promise to revolutionize a number of medical treatments. However, for some, these advances would increase social inequalities and would offer corporations, hackers, and governments the opportunity to exploit and manipulate human mind at will. For this reason, it has been suggested over the last years that any type of neurotechnology should be accompanied by a normative support aiming at protecting privacy, identity, agency, and equity of human beings. This legal support has been recently named as neurorights. In this article, we focus on we question about whether it is necessary to legislate about neurorights in this moment of 
the development of current research in biotechnology. The article does not consist of an analysis of the bill currently under development, but rather a reflection on the very idea of legislating in this area.

KEYWORDS Neurotechnology, neurorights, privacy, agency, neuroprotection.

\section{Distinciones preliminares acerca de lo mental y lo cerebral}

El concepto de neuroderecho genera más preguntas que certezas y, dado su reciente origen, la mayoría de estas preguntas se encuentran sin resolver. Para poder siquiera comenzar a entender la complejidad de la discusión, necesitamos algunas distinciones fundamentales respecto de aquello que pretende proteger y, así, diferenciar lo mental (o síquico) de lo puramente cerebral. El concepto de neuroderecho emerge, justamente, en la relación entre estos dos aspectos de la dimensión humana. Durante las últimas décadas, el debate asociado a la relación entre aquello que llamamos «lo mental» y ese complejo órgano que reside en nuestro cráneo, el cerebro, ha trascendido el contexto de lo filosófico para instalarse en la agenda de las actuales ciencias médicas experimentales. Ahora bien, en ambos contextos, el debate parece ser inteligible solo si respetamos una intuición básica sobre nuestra vida diaria, a saber, que lo mental y lo cerebral (o lo físico) poseen propiedades que los hacen fenómenos distintos, al menos, en principio (Balog, 1999; Chalmers, 1996; Jackson, 1982; Nagel, 1974).

Este problema, el cual ha sido denominado «el problema mente-cuerpo» dentro de la filosofía de la mente, consiste en encontrar la correcta enunciación de la relación entre propiedades físicas y propiedades mentales asociadas a estados subjetivos (Priest, 200o). Las propiedades físicas serían todas aquellas que pueden ser observables por un tercero y que pueden ser medidas de alguna forma. La presencia de tales propiedades en el mundo no dependería de que ocurran o no en la mente de un observador; siempre están disponibles para ser capturadas por percepción directa o por medio de instrumentos complementarios, como telescopios, microscopios, termómetros, o cualquier artefacto que nos ayude a medir una propiedad específica. ${ }^{1}$

Por otra parte, las propiedades mentales no son observables por terceros, no son de acceso público y, para algunos, incluso serían incuantificables (lo cual generaría una importante brecha explicativa para la ciencia). Este tipo de propiedades son sujeto-dependientes, ya que por necesidad ocurren en un sujeto y están exclusivamente asociadas a la experiencia consciente que un sujeto posee. En este sentido, a las propiedades mentales se les denomina privadas, porque son accedidas de forma única

1. Algunos ejemplos de este tipo de propiedades son la estructura química, la estructura molecular, forma, color, tamaño y localización espaciotemporal, entre muchas otras. 
y privada por el sujeto que experimenta este estado mental específico. Por ejemplo, cuando un sujeto $\mathrm{P}$ siente dolor, uno puede deducir de su conducta y de lo que dice que $\mathrm{P}$ siente dolor (formas de acceso en tercera persona a propiedades públicas). Es más, podríamos observar qué es lo que ocurre en su cerebro y, tal vez, eso coincidiría con la actividad eléctrica paradigmáticamente asociada a experiencias de dolor. Este tipo de información parece ser de acceso público. Sin embargo, uno no puede acceder en forma directa al dolor de P. Él tiene acceso privilegiado en primera persona a su propio dolor, y si tal dolor no ocurriese en $\mathrm{P}$, esa experiencia de dolor no podría siquiera existir. Pues bien, una de las diferencias fundamentales entre estos dos tipos de propiedades es el acceso que tenemos a ellas. Mientras el acceso a las propiedades físicas es público y sujeto-independiente, el acceso a los estados mentales es privado y sujeto-dependiente (Jackson, 1986).

Ahora, en el acontecer de cualquier estado mental conviven propiedades físicas y propiedades mentales (Jackson, 1986). Por ejemplo, mi experiencia del dolor está compuesta por propiedades físicas como la actividad de neuronas tipo-C, la acción de ciertos nociceptores en mi cuerpo, un tipo específico de procesamiento eléctricocerebral, entre muchas otras. Además, mi dolor posee sensaciones de privacidad (la sensación de que los otros no pueden acceder a mi dolor en la forma en que yo accedo a él), inefabilidad (la sensación de que las palabras muchas veces no logran capturar la complejidad de una experiencia consciente), y carácter fenoménico (la sensación de que hay una forma específica de sentir cierto estado mental), entre otras. En este contexto, es poco controversial pensar que para cada fenómeno mental debiese existir una base neurobiológica. Cuando pienso en X, algo específico debe estar sucediendo en mi cerebro que es, de alguna forma, diferente de lo que ocurre cuando pienso en Y. Esto no tiene mucho de problemático. El verdadero desafío implica establecer de forma clara el tipo de relación entre el comportamiento del cerebro y los fenómenos mentales, o, para muchos, cómo lo cerebral causa lo mental.

Sin embargo, hasta la fecha no es del todo claro cómo la actividad del cerebro está causalmente relacionada con todas las propiedades de lo mental. Para algunos, los estados mentales y los estados cerebrales son simplemente lo mismo y cualquier aparente diferencia cualitativa -o incluso ontológica- emerge de la adopción de absurdas ideas desde el sentido común (Churchland, 1984) o de ilusiones lingüísticas (Rorty, 1970). Sin embargo, posiciones así de radicales parecen no ser coherentes con nuestras intuiciones más profundas acerca de nuestra vida mental (Nagel, 2013). Para otros, si bien todo lo que existe en el universo tiene una naturaleza física, hay al menos dos formas de hablar sobre esta, una forma física y una forma mental. Para Davidson (1971), el asunto clave es que los enunciados mentales simplemente no pueden ser reducidos a enunciados físicos sin que en este juego se pierdan las características fundamentales de lo mental. En una línea no reduccionista similar, algunos autores han propuesto que, si bien todo lo que existe en el universo es físico, cuando lo físico 
(orgánico) se organiza de formas específicas, permite la emergencia de lo mental. Cuando ocurre esto, el ámbito de lo mental gozará de características fundamentales exclusivas que no pueden ser reducidas a la actividad del cerebro (Chalmers, 1996).

No es nuestra intención ofrecer una respuesta al problema mente-cuerpo acá, sin duda no nos consideramos aún capaces de aquello. Nuestro objetivo es enfatizar dos ideas. Primero, que el desafío explicativo de establecer una relación causal entre lo cerebral y lo mental está lejos de ser resuelto, y ambos ámbitos parecen ser distintos, al menos en principio. Segundo, y más importante para nuestro análisis, que la clarificación de la relación entre lo cerebral y lo mental no es un mero ejercicio retórico. Para muchos científicos, mientras mejor podamos describir esta relación, mayor conocimiento podremos alcanzar de las bases neurobiológicas de los estados mentales y, por consiguiente, podríamos comprender varias de las alteraciones médicas que afectan la funcionalidad y calidad de vida de muchas personas en el mundo. Por lo tanto, el problema mente-cuerpo no es un simple ejercicio retórico, sino que también constituye un desafío médico con importantes repercusiones en el desarrollo de la sociedad. El resto de este artículo tratará sobre un nuevo desafío filosófico y jurídico que emerge de los desarrollos médicos y científicos asociados al esclarecimiento de esta relación.

\section{Conectando lo cerebral y lo mental: Neurotecnologías y la preocupación por los neuroderechos}

Con el fin de clarificar la relación entre lo mental y lo cerebral desde un punto de vista científico, el Brain Activity Map Project (BAMP) busca la forma de desarrollar neurotecnologías que pretenden generar una nueva y revolucionaria radiografía de la forma en que la actividad eléctrica del cerebro produce (en tiempo real) fenómenos mentales como la percepción, la acción voluntaria, las memorias, los pensamientos y la conciencia (Alivisatos y otros, 2013). El BAMP pertenece a The Brain Initiative, megaproyecto que busca mapear con una especificidad sin precedente las rutas neuroeléctricas específicas a la base de nuestra vida mental (Alivisatos y otros, 2013; Andrews y Weiss, 2012). La idea de esta iniciativa no es simplemente dilucidar qué partes del cerebro estarían implicadas en la producción de ciertos estados mentales, sino cuáles son las redes neuronales específicas que generan tales estados. Pensemos en nuestra experiencia de ver una pantalla de computador. Esta experiencia consciente posee todo un aparataje neuronal que la hace posible. La idea del proyecto es trazar un mapa de cada circuito y neurona que permite mi experiencia de estar viendo la pantalla, y lo mismo para todos nuestros estados conscientes.

A simple vista, las repercusiones éticas sociales de las neurotecnologías derivadas de este proyecto son de alta consideración. Imaginemos el siguiente caso. El BAMP ha logrado diseñar un mapa de las vías neuroeléctricas específicas asociadas a los 
movimientos corporales. El — por así llamarlo- «motor-BAMP» es capaz de registrar en un disco duro todos los circuitos, potenciales de acción, recorridos específicos, parámetros homeostáticos y más asociados a la acción de tomar un cuchillo y enterrarlo en algo o alguien. Si asumimos que nuestros cerebros son similares, y que este trazado muestra cómo se instancia este tipo de movimiento en todos los humanos, quien posea la base de datos del motor-BAMP podría codificar esos impulsos con la información eléctrica codificada «enterrar cuchillo en algo o alguien» y, por medio de la transmisión mediante un artefacto que genere impulsos eléctricos, sería capaz, en efecto, de hacer que una persona acuchillase a otra.

Si bien esta caricatura es digna de una película de ciencia ficción, en realidad el escenario podría estar más cerca de lo que creemos (Powell y otros, 2013; Ienca y Haselager, 2016). En la actualidad, la mayoría de los componentes de esta sociedad interactuamos con artefactos digitales como computadores, teléfonos, relojes inteligentes, tabletas, etcétera. Estos artefactos funcionan sobre la base de impulsos eléctricos, y no sería difícil incorporar un transmisor de impulsos eléctricos por tacto que sean imperceptibles. Si el motor-BAMP se lograse construir (que es la promesa del BAMP en general), no suena descabellada la hipótesis de la programación neuroeléctrica por medio de artefactos digitales.

Ahora, si bien las aplicaciones médicas parecen ser la prioridad del BAMP (Yuste y otros, 2017), el proyecto ha abierto una serie de preocupaciones éticas en el mundo, a la luz de potenciales aplicaciones comerciales de las neurotecnologías. Tal como Alivisatos y otros (2013) sugieren, el BAMP proveerá un puente que permitirá el registro y manipulación de la actividad de circuitos, redes y eventualmente neuronas con precisión individual. Si bien existe un interés primordial en las aplicaciones médicas de las neurotecnologías, es ingenuo pensar que grandes compañías no buscarían la forma de generar aplicaciones comerciales (Fernández y otros, 2015). Gigantes como Google y Microsoft ha invertido grandes sumas en el proyecto. ${ }^{2}$ A esto se le suma el apoyo directo del Gobierno de Estados Unidos. En este contexto, ¿quién podría evitar el potencial desarrollo de aplicaciones militares basadas en neurotecnologías?

Por esta razón, The Morningside Group (un grupo de científicos asociado al BAMP) ha sugerido durante los últimos años que los avances en neurotecnologías podrían revolucionar el tratamiento de muchas condiciones médicas, pero también podrían exacerbar la inequidad social y ofrecer a corporaciones, hackers y Gobiernos internacionales la oportunidad de explotar y manipular a mucha gente (Yuste y otros, 2017: 160). A su vez, el Morningside Group ha indicado que las directrices legales y éticas actuales parecen ser insuficientes a la luz de la naturaleza de la información que el BAMP pretende registrar y manipular. Por lo tanto, se ha sugerido que el desarrollo

2. Dana Smith, «Why spend a billion dollars to map the human brain?», The Atlantic, 3 de abril de 2013, disponible en bit.ly/3pV8jii. 
de cualquier tipo de neurotecnología debería estar acompañado de un suporte normativo que tuviese como objetivo la protección de la privacidad, identidad, agencia y equidad de las personas (Yuste y otros, 2017: 159). A este soporte legal se le ha denominado recientemente neuroderechos.

El concepto de neuroderecho surge del intento de la Brain Initiative de conectar lo cerebral con lo mental mediante el trazado de un mapa de los circuitos y redes neuronales específicos que generarían nuestra actividad consciente. Esto parece ser una prueba fehaciente de que el problema mente-cuerpo no es un mero ejercicio retórico. A la luz del actual desarrollo de neurotecnologías y la masificación de artefactos digitales en nuestras rutinas diarias, no es descabellado pensar que nos estamos acercando a escenarios en que el control mental podría ser real. Según The Morningside Group, el concepto de neuroderecho pretende proteger el ámbito de lo mental. Sin embargo, hasta la fecha, este grupo aún no ha incorporado a la discusión un concepto claro de lo que entienden por mental. Según lo revisado en la primera sección, y junto a un grupo extenso de filósofos de la mente, creemos que el aspecto fenoménico de la experiencia mental humana es impenetrable desde el punto de vista científico. Pero, a la vez, creemos que la experiencia privada posee una serie de propiedades públicas que posibilitan su emergencia. Por lo tanto, consideramos que el concepto de neuroderecho hace, en realidad, referencia a la protección de las propiedades públicas de la experiencia mental humana.

Ahora bien, el único esfuerzo concreto en sistematizar los elementos del concepto establece cuatro preocupaciones fundamentales que actuarían como base para la creación de ciertos neuroderechos específicos (Yuste y otros, 2017). Primero, la preocupación por la privacidad de lo mental y el consentimiento informado refieren a la idea de que cada persona debería tener el derecho fundamental de mantener su información neuroeléctrica segura y privada, a su vez, manteniendo la opción de compartirla cuando estime conveniente. Segundo, la preocupación por la agencia y la identidad refiere a la idea de que la persona debería tener el derecho a preservar estas propiedades a la luz de potenciales cambios que podrían ser producto del uso de neurotecnologías. Tercero, existe una preocupación por la posibilidad de inequidades asociadas las mejoras cognitivas y físicas asociadas al uso de neurotecnologías. Sin embargo, no es claro cómo tal preocupación podría ser cristalizada en un derecho específico. Por último, existiría una preocupación por la forma en que ciertos sesgos podrían ser reproducidos por la programación de ciertas neurotecnologías. Sin embargo, tampoco es claro cómo tal preocupación podría expresarse en un derecho específico. 


\section{¿Es necesario legislar sobre neuroderechos?}

Fue más o menos a principios del siglo XXI que la combinación de neurociencias y derecho comenzó a producirse en los círculos académicos (Gazzinaga, 2008). Se suele sostener que el cerebro humano es, sin embargo, el objeto más complejo del universo, con cientos de billones de células nerviosas concentradas en un kilo y doscientos gramos. La magnitud de la complejidad de nuestro objeto crítico augura que las neurociencias pueden sin duda contribuir en forma significativa a la ciencia jurídica y a la práctica del derecho, pero también que dicha contribución será escasa y modesta en el futuro cercano (Morse, 2011).

Una cosa es realizar un análisis científico y doctrinal de un tema, y otra distinta es pasar a su configuración jurídica. La consideración científica toma en cuenta diversos puntos de vista disciplinarios, aunque siga un objeto formal específico. Entra en detalles, se abre a abordar el problema en sus diversos matices, discute críticamente opiniones contrapuestas, se plantea objeciones y pasa revista al desarrollo histórico del problema. La perspectiva epistemológica, en consecuencia, aborda el problema de manera integral, aunque se trate de un trabajo monográfico, y dispone de la plasticidad adecuada a los intereses del investigador y a las conclusiones a las que espera llegar. Un documento científico es un diálogo, una forma articulada de conversación que dispone de muchas herramientas para configurar sus argumentos, y se inscribe dentro de un proceso dialéctico en el que una comunidad de expertos toma pie de él para alcanzar otras razones, que salen a la luz gracias a la posibilidad de esta naturaleza discursiva. Esto anuncia su carácter permanentemente provisorio, sin que ello afecte la naturaleza del debate ni las conclusiones a las que se arribe.

Un documento jurídico, en cambio, es una prescripción normativa destinada a generar una conducta o una omisión de una conducta (Kelsen, 1994), y a la que va adjunta una promulgación y una sanción prevista para el caso de incumplimiento. Dispone de un operador deóntico, que se refiere al fin de la norma, o más bien a su propósito, si va a ser de mandato, prohibición, obligación de hacer o no hacer, o una permisión (Nino, 2003). Además, posee un contenido o acción deóntica, que no se refiere al propósito mismo, sino a lo que lleva dentro de ese propósito normativo, es decir, aquello que se incluye dentro del mencionado mandato, prohibición u obligación. Las normas jurídicas tienen por objeto hacer justicia y asegurar la certeza para las relaciones entre los miembros de la comunidad. Así, el discurso normativo debe ser preciso, parco y claro, porque su objetivo no es directamente la verdad, sino el bien común, que requiere de definiciones operativas y capaces de producir las conductas o las omisiones necesarias para la convivencia de una comunidad determinada en un momento determinado. Por esto se dice que la producción normativa requiere de exigencias técnicas y estándares disciplinarios y sistemáticos que no se 
pueden soslayar: el lenguaje del derecho no es ni puede ser el mismo que el de la discusión científica.

Ante la ausencia de una legislación específica en neuroderechos en el contexto mundial, durante los últimos dos años The Morningside Group ha contactado a legisladores y académicos en Chile para impulsar la creación del primer proyecto de ley que asegura la protección de neuroderechos a nivel mundial. ${ }^{3}$ En la actualidad existen en Chile dos iniciativas vinculadas al tema: el primero es un proyecto de reforma constitucional que modifica el artículo 19, número 1 de la Carta Fundamental, para proteger la integridad y la indemnidad mental con relación al avance de las neurotecnologías (Boletín 13.827-19), en el que se afirma que

la integridad física y síquica permite a las personas gozar plenamente de su identidad individual y de su libertad. Ninguna autoridad o individuo podrá, por medio de cualquier mecanismo tecnológico, aumentar, disminuir o perturbar dicha integridad individual sin el debido consentimiento.

Además, existe un proyecto sobre protección de los neuroderechos y la integridad mental, y el desarrollo de la investigación y las neurotecnologías (Boletín 13.828-19), el cual:

Prohíbe cualquier intromisión o forma de intervención de conexiones neuronales o intrusión o a nivel cerebral mediante el uso de neurotecnología, interfaz cerebro computadora o cualquier otro sistema o dispositivo, que no tenga el consentimiento libre, expreso e informado, de la persona o usuario del dispositivo, inclusive en circunstancias médicas. Prohíbe cualquier sistema o dispositivo, ya sea de neurotecnología, interfaz cerebro computadora u otro, cuya finalidad sea acceder o manipular la actividad neuronal, de forma invasiva o no invasiva, si puede dañar la continuidad sicológica y síquica de la persona, es decir, su identidad individual, o si disminuya o daña la autonomía de su voluntad o capacidad de toma de decisión en libertad. ${ }^{4}$

Precisamente porque se trata de una cuestión en desarrollo, parece oportuno avanzar algunas consideraciones sobre el particular que pueden ayudar a la hora de proponer un texto normativo en torno a una cuestión tan importante. Nuestro objetivo en este apartado es explorar una cuestión fundamental que surge al analizar el asunto que nos convoca, a saber, si es realmente necesario legislar sobre neuroderechos en

3. «Guido Girardi y neurocientífico español Rafael Yuste presentarán reforma constitucional para proteger los "neuroderechos"», El Mostrador, 2 de octubre de 2019, disponible en bit.ly/35jyKVn.

4. Para un análisis crítico de estos proyectos en concreto, véase Alejandra Zúñiga, Luis Villavicencio y Ricardo Salas, «¿Neuroderechos? Razones para no legislar», Ciper Académico, 11 de diciembre de 2020, disponible en bit.ly/3giElS4. Véase también Pablo López-Silva, «Ley de neuroderechos, el concepto de la mente y el escenario de la investigación en neurociencias», El Mostrador, 8 de junio de 2021, disponible en bit.ly/2RXSM4z. 
este momento y a estas alturas del desarrollo de la investigación biotecnológica, o si por el contrario su protección ya se encuentra implícita en la normativa vigente.

\section{¿Es necesario modificar la Constitución para alcanzar la protección de los neuroderechos?}

En principio, nos parece que tal modificación no sería imprescindible, por las razones que a continuación se exponen. Las garantías fundamentales consagradas por la Constitución no son meros preceptos o reglas: son principios jurídicos, focos u horizontes de significado, que contienen un conjunto de normas no necesariamente explicitadas (Alexy, 1993, 1997; Dworkin, 2002; Zagrebelsky, 2011). Hoy existe cierto consenso en que los derechos fundamentales incorporados en las constituciones tienen carácter normativo (García de Enterría, 1981), lo que afecta a todo su contenido esencial. La noción de contenido esencial pretende que el desarrollo de un derecho no destruya su contenido material, convirtiéndolo en una atribución meramente formal $y$, por lo tanto, que todo lo contenido en ese núcleo pueda ser protegido. Su referente histórico es el artículo 19, número 2 de la Constitución alemana de 1949. Del mismo modo, el artículo 17 del Convenio Europeo de Derechos Humanos prohíbe cualquier acto que pueda llevar a la destrucción de los derechos fundamentales. El Tribunal Constitucional español, por su parte, se hizo cargo de esta cuestión en una sentencia de 1981, al declarar que configura el contenido esencial de un derecho «aquellas facultades o posibilidades de actuación necesarias para que el derecho sea reconocible como pertinente al tipo descrito y sin las cuales deja de pertenecer a ese tipo».5

En el caso chileno, el artículo 19, número 26 de la Constitución sostiene que se garantiza a todas las personas:

La seguridad de que los preceptos legales que por mandato de la Constitución regulen o complementen las garantías que esta establece o que las limiten en los casos en que ello lo autoriza, no podrán afectar los derechos en su esencia, ni imponer condiciones, tributos o requisitos que impidan su libre ejercicio.

Esto debe vincularse con el artículo 5, inciso segundo, el cual señala que «la soberanía tiene como límite los derechos esenciales que emanan de la naturaleza humana», con lo cual, «el límite del contenido de los derechos se proyecta no solo al legislador, sino también al poder constituyente instituido, estableciendo una garantía de irreversibilidad en materia de derechos fundamentales, lo que nos sitúa en la materia, en una afirmación y garantía mayor de los derechos fundamentales que los tres países antes mencionados». A su vez, el Tribunal Constitucional ha indicado que «un derecho es afectado en su "esencia" cuando se le priva de aquello que le es consustan-

5. Sentencia del Tribunal Constitucional de España, 8 de abril de 1981, fundamento jurídico 8, párr. 1. 
cial de manera tal que deja de ser reconocible y que se "impide el libre ejercicio" en aquellos casos en que el legislador lo somete a exigencias que lo hacen irrealizable, lo entraban más allá de lo razonable o lo privan de tutela jurídica». ${ }^{6}$

La protección constitucional de intereses vinculados con la intimidad y la inviolabilidad de las comunicaciones - el «núcleo esencial» antes mencionado- se encuentra consagrada en el artículo 19, número 4, el cual dispone lo siguiente:

La Constitución asegura a todas las personas el respeto y protección de la vida privada y a la honra de la persona y su familia, y asimismo, la protección de datos personales. El tratamiento y protección de estos datos se efectuará en la forma y condiciones que determine la ley.

Se recoge asíla modificación por la Ley 21.096, del 16 de junio de 2018, cuyo mérito fue otorgar explícitamente rango constitucional a la protección de datos personales en Chile, como ya ha ocurrido en un número importante de países.7 Antes de este reconocimiento expreso, el resguardo de los datos personales se consideraba incorporado dentro del respeto y protección de la vida privada que consagra el mismo artículo 19, número 4 de la Constitución. Al agregarse por separado, puede interpretarse como un derecho independiente del derecho a la vida privada (Contreras, 2020). El constituyente crea para la protección de datos una reserva legal especial frente a la general establecida en el artículo 19, número 26, por la que su tratamiento y protección se convierte en materia de ley. Con anterioridad habían sido definidos normativamente los datos personales, al considerarlos como aquellos «relativos a cualquier información concerniente a personas naturales, identificadas o identificables» (Ley 19.628, artículo 2).

Resulta llamativo, además, que la reforma constitucional omita el término respeto en lo tocante a los datos personales, concediendo al parecer solo su «protección». Esta redacción no parece tener sin embargo un sentido determinante, más allá de la mera técnica legislativa (Álvarez Valenzuela, 2020). La modificación es fruto del consenso en torno a la necesidad de incorporar un régimen moderno de protección de datos personales que, a la vez, incremente la protección real de los derechos y tenga la flexibilidad necesaria que requieren los actuales flujos y volúmenes de información (Álvarez Valenzuela, 2019). El texto encarna también una dimensión normativa de reformulación conceptual del derecho a la intimidad, fruto de la irrupción de la informática y las telecomunicaciones en la vida cotidiana. La expresión autodeter-

6. Sentencia del Tribunal Constitucional de Chile, rol 43, 24 de febrero de 1987, considerando vigésimo primero.

7. El entonces presidente del Consejo para la Transparencia, Marcelo Drago, declaró que «la aprobación de la Reforma Constitucional que consagra el derecho a la protección de datos personales es un salto a la modernidad», Consejo para la Transparencia, 16 de mayo de 2018, disponible en bit.ly/35cLUn4. 
minación informativa aplicada a la protección de datos personales resalta la potestad que tiene cada cual para decir cuánto de sí mismo, de sus pensamientos, sentimientos y hechos de su vida personal se encuentra dispuesto a compartir con otros (Cerda, 2003).

Esta perspectiva positiva contenida en la idea de autodeterminación informati$v a-\mathrm{y}$ no solo negativa, como sugiere la noción de protección- abre un horizonte de significado teórico y normativo mucho más sustancioso, al enfocar el problema desde el punto de vista de las facultades del interesado en torno al almacenamiento, procesamiento y suministro de datos personales. Más allá de esta distinción, que no compete tratar aquí en profundidad, lo relevante es que el contenido del derecho aludido se encuentra formado por los distintos instrumentos que integran la protección de los datos personales, y que tal derecho tiene un núcleo indisponible, que es el propio de la garantía fundamental (Murillo de la Cueva, 1999).

¿Qué ocurre en este contexto con la iniciativa de incorporar, de modo adicional a la protección de los datos personales, el resguardo específico de los neuroderechos? En principio nos parece que, al ser estos últimos a la postre un tipo específico de datos personales, la garantía expresamente incorporada por la modificación constitucional de la Ley 21.096 tutelaría de manera suficiente este género de información privada, sin que fuera necesario agregar a la Carta Fundamental un desglose particular. En otros términos, parece razonable considerar que del núcleo normativo del artículo 19, número 4 en su versión hoy vigente queda clara la protección de todo dato personal, independiente del soporte en el que este se encuentre.

Quienes están a favor de su explícita inclusión constitucional argumentan que la cuestión tiene dos sentidos: i) en las sociedades desarrolladas científica y tecnológicamente, la posibilidad de reunir, guardar, reproducir, vincular y transmitir todo tipo de información permite que todos los actores - tanto el Estado como los particulares- tengan acceso a una gran cantidad de conocimiento, lo que puede producir perjuicios a la comunidad y a terceros mediante el aprovechamiento indebido de tales datos. Además, ii) dado el carácter potestativo de la información, la concentración de estas tecnologías en el poder público podría suponer nuevos desafíos al constitucionalismo, al igual que ocurrió con los derechos humanos de primera generación: sería necesario limitar el poder político. Esto es lo que se denomina problemas estructurales (Solove, 2006: 487), y sería el argumento para incorporar constitucionalmente elementos como los neuroderechos.

En contra de este argumento, además de lo ya indicado, hay que tener presente que las garantías constitucionales disponen de cláusula de eternidad (Ewigkeitsklausel), por lo que no pueden ser modificadas ni siquiera por el cien por ciento de los parlamentarios (Peter Häberle, citado en Ferreyra, 2009). Constituyen además un catálogo abierto desde un punto de vista hermenéutico, como ocurre por regla general con la afirmación de derechos: por su propia naturaleza consideran la posibilidad 
de nuevas atribuciones y deberes que se desprendan de los grandes focos de significado que constituyen sus diversos numerales. Los catálogos abiertos se enriquecen a través de la interpretación de las normas ya existentes. En este contexto, se podría decir que los neuroderechos son subsumibles en las garantías fundamentales que ya existen; en especial en la protección de los datos personales que se incorporó en 2005. Esto implicaría afirmar que los problemas que se presenten en el futuro en relación con la subespecie de datos personales (sobre los que recaerían los neuroderechos) ya estarían protegidos por la Constitución, porque se encuentran contenidos en la respectiva garantía fundamental antes mencionada.

La configuración formal de los derechos no enumerados se encuentra en el Bill of Rights (1791), que incorpora en la Constitución norteamericana la novena enmienda, la cual dispone que «la enumeración de ciertos derechos en la Constitución no será interpretada como la negación o el menoscabo de otros retenidos por el pueblo». El objetivo de esta norma era limitar la intervención del poder federal. En 1965, el juez Douglas declaró la inconstitucionalidad de una ley que prohibía el uso de anticonceptivos, al afirmar que «las garantías expresas de la Declaración de derechos tienen zonas de penumbra, formadas a partir de las proyecciones emanadas de las mismas, y que nos ayudan a darles vida y sustancia». ${ }^{8}$ Esto habría conducido a la Constitución a encontrarse en un estado de «benevolente descuido» (Corwin, 1978).

La oposición entre derechos «formales» que constan en la Constitución y otros no escritos que se encontrarían implícitos parece ser una interpretación forzada, por cuanto los derechos implícitos estarían ya contenidos en el texto constitucional. Lo en verdad decisivo no sería la referencia textual propiamente tal, sino su reinterpretación jurisprudencial y la construcción que la sentencia produce en cada caso (Dworkin, 1996). Se trataría por lo tanto de un proceso de reconocimiento. Se ha argumentado que este modo de razonar sería exclusivamente iusnaturalista, ya sea en la versión clásica del realismo jurídico de los autores medievales, o del racionalista de la modernidad (Carpio, 200o). Esto puede ser así cuando la norma constitucional se refiere a los derechos no enunciados como inherentes al ser humano. En otros supuestos, sin embargo, podría no tratarse de una apelación a un determinado fundamento en sentido ontológico, sino a otros argumentos, como el origen popular del poder político, las bases teóricas de las constituciones democráticas liberales o el régimen representativo. En cualquier caso, la utilidad de este criterio ha encontrado distinguidos adeptos en el constitucionalismo hispanoamericano:

Hay constituciones con una norma expresa sobre los derechos no enumerados o implícitos a los que no se puede negar ni desconocer constitucionalmente por el hecho de estar ausente del catálogo declarativo, que no debe recibir el carácter taxativo. Que-

8. Caso Griswold con Connecticut, Corte Suprema de Estados Unidos, 381 U.S. 479, 1965, 484-485. 
da la rendija de la implitud para dar entrada a otros derechos que, pacificados con los enunciados en normas expresas, tienen que disfrutar del amparo de la Constitución en un mismo nivel jerárquico con los otros y con ella misma (Bidart Campos, 1989).

Ahora bien, ¿qué sucede con la segunda forma de introducir los neuroderechos a la realidad jurídica chilena?

\section{¿Es necesaria una ley que proteja los neuroderechos?}

Si modificar la Constitución para incluir los neuroderechos parece innecesario, según hemos visto, ¿ocurre lo mismo con una ley destinada a este objeto? En este punto creemos que la cuestión es más compleja. Si bien es verdad que, en sentido estricto, basta con la protección constitucional general y el uso que hagan de ella los tribunales de justicia, nos parece, por otro lado, altamente aconsejable legislar sobre la materia que nos ocupa, por la necesidad que - a partir del marco de las garantías constitucionales de generar certeza sobre la cuestión debatida - el Poder Legislativo advierta. Dicho de otro modo: aunque en abstracto, una ley destinada a resguardar la privacidad (en un supuesto novedoso presentado por el avance de la tecnología) podría ser considerada también innecesaria en virtud de la garantía general, en la práctica un texto normativo que se refiriera directamente a este nuevo tipo de atribuciones jurídicas podría ser beneficioso para abordar la complejidad del problema, darlo a conocer en forma adecuada y generar certeza y la necesaria seguridad jurídica. Así, pues, la existencia de una ley que desarrolle los derechos y deberes asociados a los adelantos de la neurotecnología podría ser un avance para el bien común y la necesidad de justicia, siempre apreciada prudencialmente, con objeto de no dejarse arrastrar por la corriente (Morse, 2004: 82). Resuelto lo anterior, cabe preguntarse sobre las condiciones formales y materiales que debiera satisfacer semejante texto normativo, con objeto de cumplir eficazmente su objetivo.

\section{Algunas reflexiones preliminares a la hora de plantear una ley de protección de los neuroderechos}

En el resto de esta sección, quisiéramos formular algunas ideas generales que puedan ser útiles para la redacción de un proyecto de ley destinado a proteger los neuroderechos.

\section{En cuanto al objetivo de la ley}

El propósito central de una ley de protección de neuroderechos debiera ser doble: i) consagrar la protección de los «datos neuronales» que corresponden a los particulares; e ii) impedir que las tecnologías neurocientíficas puedan alterar las decisiones 
libres de dichos particulares sin su consentimiento, mediante acciones positivas destinadas a ello. Se trataría, por lo tanto, de proteger información y evitar una alteración moral externa a la conciencia. El primer objetivo se refiere al conocimiento no autorizado que terceros pueden alcanzar sobre datos neuronales personales, lo que podría acarrear un uso impropio de dicha información. El segundo apunta a la disminución de libertad que podría producirse en un individuo por la posibilidad de modificar las alternativas que se presentan a una determinada conciencia, que lo llevan a elegir una $u$ otra. Esto incluye el caso en que no debiera elegir ninguna, por inexistencia de la realidad que la causa (como si se reprodujera ante la conciencia de un sujeto la experiencia de encontrarse ante un precipicio, cuando en realidad no se ha movido de su casa).

Ambos tipos tienen dos dimensiones antijurídicas: en primer lugar, la violación de la dignidad del afectado; en segundo, la imputabilidad de las acciones ejecutadas por causa de estas intervenciones. La primera dice relación con los aspectos constitutivos de la personalidad, es decir, la capacidad de comprender abstracciones y de actuar libremente tomando como base las esencias de las cosas así conocidas. En este sentido, la libertad se entiende como la facultad de elegir entre dos o más alternativas, o bien no elegir ninguna de ellas. Elegir significa aquí tomar una decisión a partir de las alternativas racionales presentadas por la facultad que comprende, es decir, la dimensión cognitiva.

En un segundo término, surge el problema de si las acciones con efectos jurídicos que se producen por intervenciones ilícitas pueden ser imputadas, ellas y sus efectos, a los agentes. En principio, no debiera ser así, por cuanto solo se es responsable de aquello en que participan las facultades superiores. Por esto dice Tomás de Aquino (2003: q. 5, a. 3) que «el hombre es señor de su acto por el libre albedrío». Sin embargo, dada la naturaleza de las nuevas tecnologías, la perspectiva procesal-probatoria de las circunstancias de un caso de este tipo puede resultar mucho más compleja que lo acostumbrado hasta ahora, y podría ser posible que personas inocentes terminaran condenadas por delitos que solo cometieron en un sentido material, pero no formal.

Existe cierta protección normativa general de la privacidad de los datos personales que se refieren a la integridad física y sicológica, pero no en concreto a lo que ahora podemos denominar como neuroderechos. Algunos estiman que la flexibilidad hermenéutica de las normas sobre derechos humanos estaría en condiciones de proveer por sí sola la protección necesaria ante este tipo de realidades (Pariotti y Ruggiu, 2012). Una propuesta de este tipo deberá probablemente tomar como horizonte de significado normativo el texto constitucional del artículo 19, número 1, inciso primero, en el que se indica que nuestro sistema jurídico asegura a todas las personas la protección de la integridad física y síquica. En un sentido general, cualquier atentado contra la integridad del ser humano está cubierto por esta declaración y, por lo tanto, considerado en abstracto, la nueva ley sería innecesaria. Pero como el derecho no está 
compuesto solo por principios, sino también por hechos y bienes concretos, puede ser aconsejable dejar por escrito las determinaciones específicas de un cierto principio si las circunstancias, evaluadas con prudencia, así lo aconsejan (omnia patent in littera, decía Grocio). El nuevo cuerpo normativo tendría por objeto abordar un caso particular de acciones contrarias a la integridad síquica, que comprendería novedosos aspectos y posibilidades científico-tecnológicas a través de las cuales esta podría verse afectada. Dichos aspectos, si bien están contenido en el principio general de proteger la integridad síquica, la novedad y profundidad de los avances neurocientíficos, pueden hacer adecuada su consagración positiva en una ley, para mayor y mejor conocimiento de los derechos y deberes de los ciudadanos. Tal sería, en un sentido técnico jurídico, la justificación de una norma como la descrita.

\section{Evitar conceptos jurídicos confusos}

Una de las cuestiones principales que se presentará ante un proyecto legislativo de esta naturaleza será tratar de no contener nociones ambiguas que dificulten la interpretación y la aplicación de la norma. De lo explicado en el punto anterior se desprende que, por necesidad, un proyecto como el que comentamos tendrá que hacer referencia a conceptos de orden científico, biotecnológico y neurológico, con objeto de poder explicar mínimamente las situaciones, acciones u omisiones que busca reglar. Es posible que se deba hablar de privacidad de los datos neuronales, del derecho a la autonomía, libertad de decisión individual sin intervenciones farmacológicas o biotecnológicas, o cualquier fórmula que implique la participación de los nuevos conocimientos sobre el comportamiento del cerebro y de las técnicas sobre cómo intervenirlo.

Es una metodología frecuente y también recomendable que el legislador se apoye en las ciencias auxiliares para configurar las normas que requieren conocimientos técnicos de otras áreas. El carácter de auxiliar al que nos referimos aquí se configura no por causa de una supuesta inferioridad de ellas respeto de la ciencia del derecho, sino por su posición funcional a la hora de diseñar un texto jurídico: el derecho se apoya en ellas para la producción normativa, toma sus conceptos y los dota de un núcleo jurídico que permite evitar la discusión puramente científica, que haría imposible la hermenéutica de la norma a la hora de su aplicación y de su justiciabilidad. En concreto, al interior de una ley, una institución concreta debe estar claramente definida, aunque en doctrina existan diversas posturas, y la discusión académica sobre su naturaleza y efectos se prolongue sin fin. El derecho debe tomar postura para legislar, y eso implica tener la posibilidad de alcanzar algún grado mínimo de claridad sobre el tema de que se trate. La razón de esta exigencia es la certeza jurídica. Si no se fija un texto cuya interpretación sea clara, o aclarable mediante los procedimientos previstos por el derecho, la norma se convierte en inaplicable. 
La aporía que se advierte al pensar en una legislación sobre neuroderechos en el futuro inmediato es que la mayoría de las nociones que se aplican están conectadas con ideas cuyo significado es todavía ambiguo y difícil de precisar, incluso en el plano estrictamente científico. Estamos hablando de nociones como «integridad mental», «identidad individual», "privacidad mental», "continuidad sicológica», «sustratos mentales», etcétera, que tienen su correlato en el ámbito filosófico, en el que tampoco existe acuerdo sobre los modelos antropológicos que se deben utilizar para fundar los conceptos jurídicos. Todas estas fórmulas están ligadas también a la sicología, la antropología y por supuesto a la biología y a la neurociencia.

Desde un punto de vista experimental, varias de estas fórmulas lingüísticas responden todavía a modelos científicos hipotéticos, que deben ser debidamente depurados y contrastados. El tiempo de investigación, experimentación y reflexión transcurrido todavía no ha sido suficiente para alcanzar certezas sobre ellos. Sin embargo, su presencia en un cuerpo normativo se orientaría a ordenar una serie de conductas $\mathrm{u}$ omisiones que podrían tener un hondo significado en la vida de las personas. Esto resulta complejo de llevar a cabo con el estado actual del conocimiento sobre tales nociones, por cuanto no se sabe a ciencia cierta qué significan con exactitud estos conceptos.

La indeterminación mencionada entraña el riesgo de que todas estas nociones, por su incapacidad de objetivarse de modo adecuado para tener un estatus jurídico en sentido técnico, queden entregadas en los hechos exclusivamente a la decisión autónoma y subjetiva del interesado o del juez, lo que puede acarrear un sinnúmero de problemas normativos, hermenéuticos y sociales. Para poder incorporar estos conceptos con fuerza prescriptiva, y por lo tanto sancionatoria, se requiere un grado más alto de conocimiento de lo que se intenta legislar.

Lo dicho remite de nuevo a la inconveniencia de producir una norma ambigua cuando se dispone de un foco abierto de sentido, como son las garantías generales contenidas en la Constitución. Al final, podría producirse la ironía de que la ley, por no salvar este inconveniente, fuera un marco de tal naturaleza que impidiera hacer justicia, incluso tomando por base el texto constitucional. Esta insuficiencia de las nociones en comento no es comparable a la de los conceptos jurídicos indeterminados, como podrían ser las garantías constitucionales o derechos fundamentales, por cuanto estos pueden estar indeterminados de hecho, pero son esencialmente determinables de suyo. En este caso, las nociones utilizadas en el proyecto no permiten determinar con precisión qué contienen. Ninguna ley debiera contemplar conceptos incapaces de ser determinados, porque no ofrecen claridad sobre lo que se quiere proteger ni lo que se intenta prohibir. 


\section{Sobre el problema del acceso a las nuevas tecnologías neurocientíficas}

La cuestión de la igualdad a la hora de participar sin discriminaciones arbitrarias a aquellas neurotecnologías que conlleven el aumento de las capacidades síquicas deviene un debate fundamental. De hecho, uno de los mayores y más desafiantes problemas creados por las mejoras biomédicas es cómo debieran ser distribuidas (Mahlman, 2009: 218). Hay autores que defienden la tesis de que las tecnologías de enhancement o mejoras debieran poder ofrecerse sin restricciones a los ciudadanos (Swindells, 2014: 215). Conviene hacer presente que la preocupación por la desigualdad en el acceso a los procedimientos neurológicos lleva implícita la idea de que estas intervenciones generan beneficios (Bostrom y Sandberg, 2009: 329), ya que, si no fuera así, lamentarse por ello sería equivalente a protestar porque las porciones de un alimento contaminado son muy pequeñas (Kass, 2003). El fundamento último de aquellos que abogan por el acceso universal viene a ser la existencia de la obligación moral de mejorar a las personas, pues en la medida en que intervenciones específicas promueven el bienestar de los sujetos, estas constituirían la esencia de lo que resulta necesario para una buena vida (Johnston, Bishop y Trotter, 2018). Siguiendo estos presupuestos, los partidarios de esta argumentación consideran que toda la población, incluidos probablemente niños, debieran estar en condiciones de acceder a las mejoras biotecnológicas y neurológicas de un modo igualitario, sin discriminación (arbitraria) alguna, y aunque ello suponga financiamiento estatal. Aún más, se argumenta en algunos casos que las mejoras debieran ser obligatorias, pues de otro modo se atentaría contra los derechos humanos (Goold, 2017: 260).

Por otro lado, los llamados bioconservadores, es decir, aquellos que consideran que el sujeto humano no debe alterar su biología ni la condición humana de manera sustancial, están en contra de la ejecución de las mejoras radicales y, por supuesto, de su masificación (Fukuyama, 2002: 183). El argumento apunta a los posibles efectos preterintencionales que se podrían producir al pretender doblegar la naturaleza humana más allá de una finalidad de restablecimiento del orden interno (es decir, terapéutica). Las mejoras radicales se encuentran además fuera del rango propio de la experiencia humana de un adulto (Agar, 2010: 15, 30) y, por lo tanto, más alejadas aún del ámbito vital de un menor de edad, respecto de los cuales habría una cierta obligación de mejorar, según autores como Bostrom o Savulescu. Si este tipo de intervenciones estuviera cubierto por una suerte de garantía universal, la protección de los niños respecto de los padres o tutores que aceptaran incluir mejoras, o la difusión de publicidad por parte de empresas que vendieran en forma interesada estos servicios con eventuales visiones interesadamente distorsionadas, con objeto de ampliar su mercado y obtener mayores ganancias, serían problemas difíciles de manejar por parte de la autoridad (Agar, 2013: 3). Volveremos sobre las mejoras radicales en el acápite siguiente, a propósito del consentimiento. 
En estas condiciones, el debate sobre la igualdad de las mejoras neurológicas adquiere una densidad que no puede ser obviada. No hay duda de que las neurotecnologías pueden ser beneficiosas para el ser humano en la lucha contra las enfermedades o aumentando algunas capacidades. Pueden también ayudar a aumentar los niveles de satisfacción personal y la productividad social y económica. El problema radicaría en que las mejoras crearían seres humanos con mayores capacidades mentales, lo que rompería la igualdad respecto de aquellos que no han podido acceder a ellas. Esto formaría castas de personas más capaces, en condiciones de producir y acumular la riqueza y el conocimiento, lo que dejaría a los demás en una posición de desventaja. Es probable que quienes sostienen esto tenderían a incluir una norma que protegiera el acceso «sin discriminaciones arbitrarias» a aquellas neurotecnologías que tuvieran como resultado el aumento de las capacidades síquicas, de modo de establecer el derecho a un «acceso igualitario» a la mejora cerebral. Esto lleva implícita la idea de que, si el poder político y jurídico no interviene, las mejoras cognitivas solo podrían ser costeadas por las personas con mayor poder adquisitivo, lo que ahondaría las diferencias entre unos y otros y propiciaría la formación de grandes dificultades sociales. Así, en virtud de las mejoras, los más pudientes serían a la vez los más capaces, lo que podría llevar a una situación arbitraria que sometería a la mayoría de los ciudadanos a un estado de vulnerabilidad (Parens, 1998: 8; Veit, 2018: 407).

Estas legítimas preocupaciones deben encontrar una concreción jurídica en una eventual ley, si tenemos presente que al ordenamiento jurídico le resulta difícil asegurar el acceso material a las nuevas tecnologías por parte de toda la población, en virtud de razones como que no se puede saber de antemano si las mejoras biotecnológicas o neurológicas que se van a ofrecer tendrán necesariamente un efecto bueno en la población, aumentando su capacidad cognitiva o moral o su bienestar, o bien si sus efectos preterintencionales negativos serán, a la postre, superiores a los beneficios. Esta es una cuestión que debe decidirse caso a caso, si es que se dispone de las herramientas para ello, pero resulta un tanto arriesgado proteger jurídicamente el acceso a ellas ex ante.

La segunda objeción se refiere a que se encuentra fuera de las posibilidades de la ley garantizar un acceso igualitario a una prestación determinada. Esto constituye una decisión prudencial del poder gubernativo, no legislativo, porque depende en realidad de las condiciones materiales concretas para el ejercicio de la justicia distributiva. Por otro lado, no resulta claro qué tipo de tecnología debiera ser protegida en su acceso igualitario y cuál no. Este límite es difícil de establecer, y en consecuencia no debiera figurar en una norma jurídica si no existen las mínimas posibilidades de precisarlo, so pena de caer en situaciones absurdas o manifiestamente injustas. En este sentido, ¿qué mejora neurocognitiva se va a incorporar a un eventual proyecto de ley: la mejora terapéutica o la mejora transhumana? (Agar, 2013: 15). Si se trata de la primera, la cuestión — la neurocognitiva - presenta menos problemas que la segun- 
da, pues es muy discutible que un cambio de naturaleza transhumana - hablamos de mejoras radicales - deba obligatoriamente ser financiado, o siquiera promovido, por el Estado. Por mejora terapéutica entendemos aquí aquellas que buscan restablecer el funcionamiento normal del ser humano, mientras que la mejora radical o transhuma$n a$ es aquella que aumenta las facultades más allá de lo que es considerado un rango normal en el ser humano (Harris, 2009, 142).

\section{Información y consentimiento}

Es razonable pensar que una ley de este tipo debiera preocuparse del consentimiento respecto de las mejoras, así como de la posible intromisión en la privacidad de la conciencia, cuando ello sea técnicamente posible. En este sentido, aquel que no presta su consentimiento fehaciente para que se le practique alguna intervención neurotecnológica, no podría ser obligado a ello. Este consentimiento debiera ser también expreso, no tácito y propio de personas jurídicamente capaces, de acuerdo con la legislación vigente.

Desde el punto de vista de la aquiescencia en las intervenciones cerebrales, también debiera considerarse el problema planteado antes, a saber, que no es posible o resulta muy dudoso prestar un consentimiento libre e informado respecto de realidades que se encuentran hasta el momento fuera del rango de la experiencia humana, como podría ocurrir en las intervenciones que mejoran atributos o cualidades síquicas significativas hasta niveles que exceden ampliamente lo que en la actualidad es posible para un miembro de nuestra especie (Agar, 2013). Así, las mejoras que hemos caracterizado como radicales conllevan el problema de que en ellas resulta muy difícil la formación de un consentimiento informado y consciente, por cuanto pertenecen a un estado (supuestamente) poshumano. Esto quiere decir que podrían, tarde o temprano, decepcionar y no producir la felicidad que se busca ex ante, al estar situadas fuera del horizonte de significado normal de conocimiento humano. Un ser humano puede imaginarse lo que significa correr veinte kilómetros más rápido de lo que compete a un hombre normal, pero no tiene manera de saber qué implica en realidad correr, por ejemplo, a quinientos kilómetros por hora. Esta última posibilidad queda por completo fuera del ámbito de su experiencia, y por lo tanto en principio no puede aceptarse ni rechazarse en sentido teórico, por lo que constituiría a la vez una irresponsabilidad en sentido práctico. En este punto, surge la pregunta de si el derecho debe permitir que un individuo tome una decisión irreversible en las condiciones que acabamos de describir.

Con todos estos antecedentes, un proyecto de ley de este tipo debiera garantizar de algún modo a los usuarios de neurotecnologías: i) la advertencia de que no se sabe bien qué se puede aceptar o rechazar, y ii) la información sobre las potenciales consecuencias negativas o efectos secundarios, así como de los posibles efectos cola- 
terales desconocidos en el tiempo presente, tanto porque no se tienen elementos para identificarlos, como porque es imposible hacerlo.

Por último, como sugerencia, podemos agregar que sería muy deseable para las áreas de investigación clínico-médica en las materias de la ley, que no se exija el consentimiento considerado por el título 5 del Código Sanitario, es decir: derecho a que se le otorgue sin costo para el paciente que participa en un ensayo clínico la continuidad del tratamiento por todo el tiempo que persista su utilidad terapéutica, conforme al protocolo de investigación respectivo (artículo 111, letra c), como tampoco la responsabilidad a todo evento por daños ocasionados por la investigación (artículo 111, letra e), dada la carga que se impone a los titulares de la investigación, y que ha probado ser contraproducente a la hora de incentivar la experimentación científica.

\section{Conclusiones}

De acuerdo con lo que se ha dicho, nos parece que si bien una modificación constitucional para incluir estos supuestos derechos neurológicos parece técnicamente innecesaria, por cuanto estaría ya contenida de un modo general la protección de su privacidad en la garantía fundamental respectiva, creemos que la presentación de un proyecto de ley sobre el tema puede ser aconsejable, con objeto de hacer conscientes a los ciudadanos de la importancia del tema y de los derechos y deberes asociados, así como resaltar la importancia de los adelantos científicos y tecnológicos de cara al bien común.

Sin embargo, un proyecto de esta naturaleza debe ser muy cauto a la hora de configurar su núcleo normativo, porque los adelantos científicos en el tema, si bien existen y se encuentran en desarrollo - como ocurre con las experiencias compartidas por el director del proyecto BRAIN durante su visita a Chile en octubre de 2019 y en enero $2020-,{ }^{9}$ estos todavía se encuentran en un estado de experimentación, de modo que el porcentaje de conocimiento todavía hipotético es alto. Esta circunstancia podría acarrear consecuencias jurídicas nefastas si se manifiesta normativamente de un modo frívolo o poco preciso.

A pesar de este riesgo, nos parece que existen ciertas cuestiones que, por su practicidad y aplicación en el corto tiempo, debieran tener una cierta forma jurídica que sirva de base para reglar el tema y para corregir la norma de acuerdo con los nuevos adelantos y descubrimientos de la neurociencia. Entre estos se hallan conceptos como la identidad e integridad del sujeto humano en el acto del pensamiento, del

9. Los autores tuvieron ocasión de integrar una mesa de trabajo con el profesor Dr. Rafael Yuste durante sus visitas en las fechas señaladas, a propósito de la posibilidad de legislar sobre la protección de los neuroderechos, en el Centro de Bioética de la Facultad de Medicina de la Pontificia Universidad Católica de Chile. 
que se deriva la propiedad de una determinada información sicológica frente a algunas eventuales técnicas neurocientíficas que podrían poner en duda la naturaleza privada de esa información, la naturaleza sicológica de la decisión libre, etcétera. A propósito de la propiedad de la información mental, es preciso tener en cuenta que la información que se contiene en soportes digitales no puede exhibir el mismo estatus de propiedad privada o excluyente que la que se ejerce sobre otro tipo de realidades presentes o derivadas del mundo corpóreo, por cuanto el soporte digital es, por su propia naturaleza, un medio que se constituye sobre la posibilidad de la transferencia de los datos. Este será el estatus que se debería llegar a proteger con una ley como la que hablamos. En el fondo, se trata de producir instrumentos normativos capaces de resguardar la información mental aunque esta se encuentre, por decirlo así, en línea.

Finalmente, nos parece que todas estas cuestiones son muy relevantes para la definición de lo humano, y requieren por lo tanto de la adopción por parte del legislador de modelos antropológicos explicativos que se aproximen a la realidad humana efectiva, sin reducirla a uno de sus aspectos con exclusión de los otros. Sería ideal que el futuro proyecto de ley, al tener en cuenta el funcionamiento de la mente y su captación mediante procedimientos informáticos, responda a la configuración del ser humano como unidad integral de corporalidad y conciencia, mente y cuerpo, cuerpo y alma, y no solo a uno de estos aspectos, como suele ocurrir en ocasiones.

\section{Referencias}

Agar, Nicholas (2010). Humanity's end. Cambridge: MIT Press.

-. (2013). Truly human enhancement: A philosophical defense of limits. Cambridge: MIT Press.

Alexy, Robert (1993). Teoría de los derechos fundamentales. Madrid: Centro de Estudios Constitucionales.

-. (1997). Teoría de la argumentación jurídica. Madrid: Centro de Estudios Constitucionales.

Alivisatos, Paul A., Miyoung Chun, George M. Church, Karl Deisseroth, John P. Donoghue, Ralph J. Greenspan, Paul L. McEuen, Michael L. Roukes, Terrence J. Sejnowski, Paul S. Weiss y Rafael Yuste (2013). «The brain activity map». Science, 339 (6.125): 1.284-1.285. DOI: 10.1126/science.1236939.

Álvarez Valenzuela, Daniel (2019). "Agenda legislativa de datos personales en Chile». Revista Chilena de Derecho y Tecnología, 8 (1): 1-2. DOI: 10.5354/0719-2584.2019.53761.

-. (2020). «La protección de datos personales en el contexto de pandemia y la constitucionalización del derecho a la autodeterminación informativa». Revista Chilena de Derecho y Tecnología, 9 (1): 1-4. DOI: 10.5354/0719-2584.2020.57777. 
Andrews, Anne y Paul Weiss (2012). «Nano in the brain: Nano-neuroscience», ACS Nano, 6 (10): 8.463-8.464. DOI: 10.1021/nn304724q.

BALOG, Katalin (1999). "Conceivability, possibility, and the mind-body problem». The Philosophical Review, 108 (4): 497-528. DOI: 10.2307/2998286.

Bidart Campos, Germán (1989). Teoría general de los derechos humanos. Ciudad de México: UNAM.

Bostrom, Nick y Anders Sandberg (2009), «Cognitive enhancement: Methods, ethics, regulatory challenges». Science and Engineering Ethics, 15 (3):311-341. DOI: 10.1007/s11948-009-9142-5.

CARPIO, Edgar (2000). «El significado de la cláusula de los derechos no enumerados». Cuestiones Constitucionales, 3: 1-25. DOI: 10.22201/iij.24484881e.2000.3.5595.

Cerda Silva, Alberto (2003). «Autodeterminación informativa y leyes sobre protección de datos». Revista Chilena de Derecho Informático, 3: 45-75. DOI: 10.5354/0717-9162.2011.10661.

Chalmers, David (1996). The conscious mind. Nueva York: Oxford University Press. Churchland, Paul (1984). Matter and consciousness. Cambridge: MIT Press.

Contreras, Pablo (2020). «El derecho a la protección de datos personales y el reconocimiento de la autodeterminación informativa en la Constitución chilena». Revista de Estudios Constitucionales, 18 (2): 87-120. DOI: 10.4067/ So718-52002020000200087.

Corwin, Edward (1978). The constitution and what it means today. Princeton: Princeton University Press.

Dworkin, Ronald (1996). Freedom's law. Oxford: Oxford University Press.

-. (2002). Los derechos en serio. Barcelona: Ariel.

DAvidson, Donald (2003). Mental events. Londres: Oxford University Press.

Fukuyama, Francis (2002). Our postmodern future: Consequences of the biotechnological revolution. Volumen 2. Nueva York: Farrar, Straus \& Giroux.

FernÁNDEZ, Álvaro, Nikhil Sriraman, Brian Gurevitz y Olivier Oiuiller (2015). Pervasive neurotechnology: A groundbreaking analysis of 10,000+ patent filings transforming medicine, health, entertainment and business. San Francisco: Sharp Brains. Disponible en bit.ly/2SvqVck.

Ferreyra, Raúl (2009). «Entrevista a Peter Häberle». Boletín Mexicano de Derecho Comparado, 126: 1.621-1.645. Disponible en bit.ly/3wvqc9U.

GARCía DE ENTERRÍA, Eduardo (1981). La Constitución como norma y el Tribunal Constitucional. Madrid: Civitas.

GazZINAGA, Michael (2008). «The law and neurosciences». Neuron, 60 (3): 412-415. DOI: 10.1016/j.neuron.2008.10.022.

GooLD, Imogen (2007). The legal aspects of cognitive enhancement: Rethinking cognitive enhancement. Oxford: Oxford University Press. 
HARrIs, John (2009). «Enhancements are a moral obligation». En Julian Savulescu y Nick Bostrom (editores), Human enhancement (pp. 131-155). Nueva York: Oxford University Press.

IENCA, Marcello y Pim Haselager (2016). «Hacking the brain: Brain-computer interfacing technology and the ethics of neurosecurity». Ethics and Information Technology, 18: 117-129. DOI: 10.1007/s10676-016-9398-9.

JACKson, Frank (1982). «Epiphenomenal qualia». The Philosophical Quarterly, 32: 127-136. DOI: 10.2307/2960077.

-. (1986). «What Mary didn't know». The Journal of Philosophy, 83 (5): 291-295. DOI: $10.2307 / 2026143$.

Johnston, Ysabel, Jeffrey P. Bishop y Trotter Griffin (2018). «The moral imperative to morally enhance». The Journal of Medicine and Philosophy, 43 (5): 485-489. DOI: 10.1093/jmp/jhyo19.

KAss, Leon (2003). «Ageless bodies, happy souls: Biotechnology and the pursuit of perfection». New Atlantis, 1: 9-18. Disponible en bit.ly/35f 31 EI.

Kelsen, Hans (1994). Teoría general de las normas. Ciudad de México: Trillas.

Mahlman, Maxwell J. (2009). The price of perfection: Individualism and society in the era of biomedical enhancement. Baltimore: The Johns Hopkins University Press.

Morse, Stephen J. (2004). «New neuroscience, old problems: Legal implications of brain science». Cerebrum, 6 (4): 81-9o. Disponible en bit.ly/3vlXIxN.

-. (2011). «Lost in translation? An essay on law and neuroscience». Law \& Neuroscience, 13: 529-562. Disponible en bit.ly/2RP5t1w.

Murillo de la Cueva, Pablo (1999). «La construcción del derecho a la autodeterminación informativa». Revista de Estudios Políticos, 104: 35-6o. Disponible en bit. ly/2TsSS4u.

NAgel, Thomas (1974). «What is it like to be a bat?». The Philosophical Review, 83 (4): 835-856. DOI: $10.2307 / 2183914$.

-. (2013). Mind and cosmos. Oxford: Oxford University Press.

Nino, Carlos Santiago (2003). Introducción al análisis del derecho. Buenos Aires: Astrea.

PARens, Erik (1998). «Is better always good? The Enhancement Project». The Hastings Center Report, 28: 1-17. DOI: 10.2307/3527981.

PARIOTTI, Elena y Daniele Ruggiu (2012). «Governing nanotechnologies in Europe: Human rights, soft law and corporate social responsibility». En H. Van Lente, C. Coenen, K. Konrad, L. Krabbenborg, C. Milburn, F. Seifert, F. Thoreau y T. Zülsdorf (editores), Little by little: Expansions of nanoscience and emerging technologies. Heidelberg: IOS Press/AKA-Verlag. Disponible en bit.ly/3goMBA2.

Powel, Courtnet, Munetomo Masaharu, Martin Schuleter y Masataka Mizukoshi (2013). «Towards thought control of next-generation wearable computing devices». En Kazayuki Imamura, Shiro Usui, Tomoaki Shirao, Takuji Kasamatsu, Lars 
Schwabe y Ning Zhong (editores), Brain and health informatics (pp. 427-438). Cham: Springer. DOI: 10.1007/978-3-319-02753-1_43.

PrIEST, Stephen (2000). The subject in question. Londres: Routledge.

RoRTy, Richard (1970). «In defense of eliminative materialism». The Review of Metaphysics, 24 (1): 112-121. Disponible en bit.ly/35kRaoJ.

Santo Tomás de Aquino (2003). De veritate 24: El libre albedrío. Volumen 165. Pamplona: Cuadernos de Anuario Filosófico, Serie Universitaria.

Solove, Daniel (2006). "A taxonomy of privacy». University of Pennsylvania Law Review, 154 (3): 477-580. Disponible en bit.ly/3vmYHhp.

SwINDELLS, Fox (2014). «Economic inequality and human enhancement technology». Humana Mente, 26: 213-222. Disponible en bit.ly/3wnMHoq.

VeIT, Walter (2018). «Cognitive enhancement and the threat of inequality». Journal of Cognitive Enhancement, 2: 404-410. DOI: 10.1007/s41465-018-0108-x.

Yuste, Rafael, Sara Goering, Philipp Kellmeyer y otros (2017). «Four ethical priorities for neurotechnologies and AI». Nature, 551 (8): 159-163. DOI: 10.1038/551159a. ZAgrebelsky, Gustavo (2011). El derecho dúctil. Madrid: Trotta.

\section{Sobre los autores}

Pablo López-Silva es doctor en Filosofía y magíster en Investigación en Filosofía por la Universidad de Manchester, Reino Unido. Profesor adjunto de la Escuela de Psicología de la Universidad de Valparaíso, Chile. Su correo electrónico es pablo. lopez.silva@gmail.com. (D) https://orcid.org/oooo-0oo1-7457-7724.

RAÚL MADRID es doctor en Derecho y magíster en Filosofía por Universidad de Navarra, España. Profesor titular ordinario de la Facultad de Derecho y profesor de la Facultad de Filosofía de la Pontificia Universidad Católica de Chile. Director del programa de Derecho, Ciencia y Tecnología de la misma Facultad de Derecho. Su correo es rmadrid@uc.cl. (D): https://orcid.org/oooo-0o03-4592-6985. 


\title{
REVISTA CHILENA DE DERECHO Y TECNOLOGÍA
}

La Revista de Chilena de Derecho y Tecnología es una publicación académica semestral del Centro de Estudios en Derecho Informático de la Facultad de Derecho de la Universidad de Chile, que tiene por objeto difundir en la comunidad jurídica los elementos necesarios para analizar y comprender los alcances y efectos que el desarrollo tecnológico y cultural han producido en la sociedad, especialmente su impacto en la ciencia jurídica.

\author{
EDITOR GENERAL \\ Daniel Álvarez Valenzuela \\ (dalvarez@derecho.uchile.cl) \\ SITIO WEB \\ rchdt.uchile.cl \\ CORREO ELECTRÓNICO \\ rchdt@derecho.uchile.cl \\ LICENCIA DE ESTE ARTÍCULO \\ Creative Commons Atribución Compartir Igual 4.o Internacional
}

\begin{abstract}
La edición de textos, el diseño editorial
y la conversión a formatos electrónicos de este artículo

estuvieron a cargo de Tipográfica

(www.tipografica.io).
\end{abstract}

